\title{
Os impactos da lei de responsabilidade fiscal nos municípios do Vale do Mucuri: um estudo aplicado a dados em painel
}

\author{
Geórgia Fernandes Barros ${ }^{1}$ \\ Luiz Souza Gomes²
}

\begin{abstract}
Resumo: O trabalho teve como objetivo avaliar os impactos de indicadores fiscais, respaldados na Lei de Responsabilidade Fiscal, sobre o resultado primário dos municípios do Vale do Mucuri, em Minas Gerais, para o período compreendido entre 2000 e 2015. A pesquisa realizada, de natureza quantitativa e descritiva, adotou modelos lineares estáticos para dados em painel. A pesquisa aponta para um baixo dinamismo na arrecadação de receitas tributárias e para um efeito negativo dos gastos com pessoal sobre o resultado primário. Além disso, assinala para um impacto maior das despesas com pessoal do que das receitas tributárias sobre o resultado primário, retratando o baixo dinamismo da gestão fiscal local.
\end{abstract}

Palavras-Chave: Lei de Responsabilidade Fiscal, Finanças Públicas, Vale do Mucuri.

Abstract: This study aims to evaluate the impact of fiscal indicators, supported by the Fiscal Responsibility Law, on the primary outcome of the municipalities of the Mucuri Valley, in Minas Gerais, for the period 2000 to 2015. The quantitative and descriptive research adopted static linear models for panel data. The results points to a low dynamism in the collection of tax revenues and to a negative effect of personnel expenses on the primary result. In addition, it points to a greater impact of personnel expenses than of tax revenues on the primary result, portraying the low dynamism of local tax management.

Keywords: Fiscal Responsibility Law, Public finances, Mucuri Valley.

\section{INTRODUÇÃ̃}

Há na literatura diversos estudos que abordam a dinâmica dos gastos do setor público e evidenciam a necessidade da gestão eficiente, adequada e transparente dos recursos públicos, tanto em âmbito federal, quanto nos âmbitos estadual e municipal.

A partir da promulgação da Lei Complementar no. 101, de 04 de maio de 2000, conhecida como Lei de Responsabilidade Fiscal (LRF), foram estabelecidas às

\footnotetext{
1 Mestre em Teoria Econômica, pela Universidade Federal do Espírito Santo, Doutora em Desenvolvimento Regional pela Universidade Federal de Minas Gerais. Professora adjunta na Universidade Federal dos Vales do Jequitinhonha e Mucuri (UFVJM). Coordenadora do Mestrado em Administração Pública da UFVJM. georgiafbarros@gmail.com.

${ }^{2}$ Mestrando em Administração Pública na Universidade Federal dos Vales do Jequitinhonha e Mucuri (UFVJM).
} 
administrações públicas de todas as esferas de governo os princípios e normas fundamentais regulamentadores do disposto nos artigos 163 a 169 da Constituição federal de $1988(\mathrm{CF} / 88)$.

Nesse sentido, a LRF assume relevante papel no tocante à gestão fiscal responsável. $\mathrm{O} \S 1^{\circ}$ do artigo $1^{\circ}$ da referida Lei, dispõe sobre a gestão fiscal planejada e transparente no intuito de prevenir riscos e corrigir desvios capazes de influenciar o equilíbrio das contas públicas, a partir do cumprimento de metas de resultados entre receitas e despesas e a subordinação a balizes previstos.

O presente trabalho tem por objetivo analisar o enquadramento das prefeituras dos 27 municípios do Vale do Mucuri, em Minas Gerais, em relação ao proposto pela LRF em termos do cumprimento de metas de resultado fiscal, nos anos 2000 a 2015, a partir da utilização de dados em painel.

O trabalho é motivado pela maior compreensão da trajetória fiscal dos municípios do Vale do Mucuri, à luz da Lei de Responsabilidade Fiscal, em especial no que tange à rigidez das despesas com pessoal e à baixa arrecadação tributária desses municípios, em geral, de pequeno porte e caracterizados pelo baixo dinamismo de suas economias e pela presença marcante de desigualdade e vulnerabilidade social.

\section{2 - REFERENCIAL TEÓRICO}

A Lei Complementar $n^{\circ}$ 101/2000 (LRF) constitui parte do sistema de gestão fiscal implantado no País e estabelece, em regime nacional, normas de finanças públicas voltadas para a responsabilidade na gestão fiscal, com amparo no Capítulo II do Título VI da Constituição Federal de 1988.

Em seu $\S 1^{\circ}$ do artigo $1^{\circ}$, a LRF estabelece que a responsabilidade na gestão fiscal deva ser uma ação planejada e transparente, a partir do cumprimento de metas de resultados entre receitas e despesas e da obediência a parâmetros previstos:

\footnotetext{
De acordo com o parágrafo $1^{\circ}$ do artigo $1^{\circ}$ da referida Lei: A responsabilidade na gestão fiscal pressupõe a ação planejada e transparente, em que se previnem riscos e corrigem desvios capazes de afetar o equilíbrio das contas públicas, mediante o cumprimento de metas de resultados entre receitas e despesas e a obediência a limites e condições no que tange a renúncia de receita, geração de despesas com pessoal, da seguridade social e outras, dívidas consolidada e mobiliária, operações de crédito, inclusive por antecipação de receita, concessão de garantia e inscrição em Restos a Pagar (BRASIL, 2000).
}

No conjunto de normas e princípios estabelecidos pela LRF, merecem destaque: 
- limites de gasto com pessoal: a lei estabelece limites para essa despesa em relação à receita corrente líquida para os três Poderes e para cada nível de governo. No âmbito municipal, a LRF determina que o gasto com pessoal não pode exceder $60 \%$ da receita corrente líquida. Desse total, o gasto do Executivo não pode superar 54\% e o gasto do Legislativo deve ficar em, no máximo, 6\%, incluindo o Tribunal de Contas do Município;

- limites para o endividamento público, estabelecidos pelo Senado Federal por proposta do Presidente da República;

- definição de metas fiscais anuais: para os três exercícios seguintes;

- mecanismos de compensação para despesas de caráter permanente, de modo que os governantes não poderão criar uma despesa continuada (por prazo superior a dois anos) sem indicar uma fonte de receita ou uma redução de outra despesa; e,

-mecanismo para controle das finanças públicas em anos de eleição: a LRF impede a contratação de operações de crédito por antecipação de receita orçamentária no último ano de mandato e impede o aumento das despesas com pessoal nos 180 dias que antecedem o final do mandato (BRASIL, 2000).

A LRF objetiva, assim, prevenir os déficits nas contas públicas, manter sob controle o nível de endividamento público, impedindo que os gestores assumam obrigações e encargos sem a correspondente fonte de receita ou a redução da despesa, e impõe a imediata correção dos desvios na conduta fiscal, com o intuito de assegurar o equilíbrio das finanças públicas (GERIGK, 2008). A LRF traz, assim, uma concepção de equilíbrio nas contas públicas, traduzidas no Resultado Primário equilibrado ((NASCIMENTO; DEBUS, 2002), (MATIAS-PEREIRA, 2006 e 2007)) .

Alguns trabalhos têm estudado a Lei de Responsabilidade Fiscal no âmbito municipal (como Botelho (2002), Sakurai (2005), Menezes (2005), Fioravante et al. (2006), Giuberti (2006), Veloso e Teixeira (2007), Santolin, Jayme Jr. e Reis (2009), dentre outros.), partindo do princípio de que os municípios têm papel fundamental na estrutura político-administrativa brasileira.

A esse respeito, Menezes (2002) e Matias e Campello (2000) apontam que o município é reconhecido como a instituição que pode levar de forma mais eficiente e eficaz a presença do Poder Público ao interior do País, além de ser agente potencializador do desenvolvimento econômico local. É no município, a menor unidade administrativa da Federação, que se apresentam as condições mais adequadas para a construção de um quadro nacional democrático e também, onde se encontram as 
condições mais favoráveis para intervenção, uma vez que o Poder Público local está mais próximo dos problemas e é suscetível a controle mais direto da sociedade.

O objetivo precípuo das administrações públicas municipais é a prestação de serviços públicos locais aos cidadãos. Com esse intuito, é utilizado por estas administrações o Sistema de Planejamento Integrado, composto pelo Plano Plurianual PPA, a Lei de Diretrizes Orçamentárias - LDO e a Lei Orçamentária Anual - LOA, mais conhecida com Orçamento Público.

No PPA são apresentadas as diretrizes, programas, com discriminação dos objetivos e ações e, estas são detalhadas em metas a serem cumpridas pelo governo (ANDRADE, 2007) e contempla o planejamento municipal para quatro anos. A LDO, por sua vez, dispõe sobre a elaboração da Lei Orçamentária Anual - LOA e abarca as metas e prioridades governamentais para determinado exercício financeiro. As diretrizes da LDO são anuais e têm por base o planejamento de médio prazo PPA. A LOA é constituída pelas ações governamentais e as metas financeiras que o ente público municipal pretende realizar no decorrer do exercício financeiro que fizer referência, atendendo o que foi definido na LDO (CARVALHO, 2010).

Com o advento da LRF, a LDO obteve maior relevância para o efetivo planejamento do comportamento das finanças públicas, e norteador da elaboração do Orçamento. Conforme se verifica a partir do art. $4^{\circ}$ da LRF, a LDO também deverá dispor sobre equilíbrio entre receitas e despesas, metas fiscais, riscos fiscais, programação financeira e cronograma de execução mensal de desembolso, critérios e formas de limitação de empenho, condições e exigências para as transferências de recursos a entidades públicas e privadas, forma de utilização e montante de reserva de contingência e integração da LOA, concessão ou ampliação de incentivo ou benefício de natureza tributária da qual decorra renúncia de receita.

No âmbito desse cenário de controle dos recursos públicos e de responsabilidade social, foram definidos percentuais para gastos com pessoal do Poder Executivo, Poder Legislativo, Poder Judiciário e Ministério Público, estimados sobre a Receita Corrente Líquida (RCL), assim como limites para a dívida consolidada líquida e para as operações de créditos. Desse modo, a despesa total com pessoal, em cada período de apuração e em cada ente da Federação, não poderá exceder os percentuais da receita corrente líquida de 50\% para a União, 60\% para Estados e municípios (BRASIL, 2000).

A Lei de Responsabilidade Fiscal, desse modo, prevê não somente a questão do equilíbrio entre as receitas e despesas, mas também a questão do endividamento, 
analisado em longo prazo. Abrange o aspecto contínuo e progressivo das mudanças mandatórias para a obtenção do equilíbrio das contas públicas, em todas as esferas de governo.

Segundo Sousa et al., (2011), quando se trata da gestão pública municipal, a perspectiva da Lei de Responsabilidade Fiscal (LRF) torna-se ainda mais relevante, uma vez que, diante da municipalização, há a escassez de recursos para investimentos e da crescente demanda por serviços sociais.

O presente estudo visa contribuir, à luz das discussões e trabalhos pertinentes à LRF e sua aplicação aos municípios, na medida em que se direciona a avaliar os impactos da Lei de Responsabilidade Fiscal no comportamento fiscal municipal do Vale do Mucuri, para os anos 2000 a 2015. Para a realização do mesmo, serão utilizados modelos estáticos de dados em painel, conforme elaboração a seguir.

\section{3 - METODOLOGIA}

Esta pesquisa caracteriza-se, quanto à abordagem do problema, de natureza de avaliação quantitativa de dados, e quanto aos objetivos, trata-se de uma pesquisa descritiva uma vez que foi realizado um levantamento documental com coleta, tratamento análise e interpretação de dados secundários.

\section{1- Dados e descrição das variáveis}

Para se avaliar o impacto da LRF sobre o desempenho fiscal do universo dos municípios do Vale do Mucuri, foram utilizadas as variáveis fiscais: receita tributária, receita corrente, despesa de pessoal e saldo orçamentário per capita. Os dados foram retirados do banco de dados Finanças do Brasil (FINBRA), disponibilizado no sitio eletrônico da Secretaria do Tesouro Nacional.

A primeira variável explicativa estimada foi a razão entre despesa de pessoal/receita corrente, a qual retrata a parcela de receitas correntes comprometidas com despesas de pessoal. A segunda variável explicativa aferida foi a razão receita tributária/receita corrente e trata-se de uma medida de independência municipal às receitas oriundas de transferências constitucionais advindas do Estado e da União (Sakurai, 2005).

Além dessas, foram estimadas duas variáveis dummies: a variável dummy1, que assume valor 1 no período 2001 em diante, e zero, caso contrário; e variável dummy2, que assume valor um especificamente no ano 2001 e zero caso contrário. A construção 
dessas variáveis se justifica pelo fato de que um número considerável de municípios não se encontrava obrigado a cumprir as disposições da LRF em 2000.

\section{2- Modelo linear para dados em painel}

O método para análise dos indicadores propostos foi à regressão de dados em painel estático, caracterizados por possuírem observações em duas dimensões que em geral são o tempo e o espaço (CAMERON \&TRIVEDI, 2005 e 2009).

Os modelos lineares estáticos de dados em painel apresentam a seguinte especificação:

$$
y_{i t}=b_{0}+b_{1} x_{i t 1}+\ldots+b_{k} x_{i t k}+u_{i t}
$$

Os dados em painel acompanham a variável dependente $\left(\mathrm{y}_{\mathrm{i}}\right)$ para os indivíduos (i), ao longo do tempo (t).

Os modelos lineares estáticos são representados pela seguinte especificação::

$$
\mathrm{y}_{\mathrm{it}}=\mathrm{x}^{\prime}{ }_{\mathrm{it}} \beta+\eta_{\mathrm{i}}+\mathrm{u}_{\mathrm{it}}
$$

Nesses casos de painéis, ๆi capta a heterogeneidade não observada ou efeitos individuais e xit pode incluir dummies de período.

Podemos especificar esse modelo da seguinte forma:

$$
\begin{gathered}
y_{i t}=x^{\prime}{ }_{i t} \beta+\varepsilon_{i t} \\
\text { sendo: } \varepsilon_{i t}=\eta_{i}+u_{i t}
\end{gathered}
$$

ou seja, incorpora um termo de erro composto $\varepsilon_{i t}$, a partir de $\eta_{i}$ (que é a parte constante entre indivíduos e o efeito individual) e $u_{i t}$ (que é o erro aleatório e segue uma distribuição normal).

Nessa especificação dos modelos lineares estáticos, quatro hipóteses são adotadas: $1^{\mathrm{a}}$ ) trabalha-se com uma amostra aleatória, com $\mathrm{N}$ relativamente grande e $\mathrm{T}$ pequeno, ou seja, assintótico em $\mathrm{N} ; 2^{\mathrm{a}}$ ) posto completo de $\mathrm{x}($ exceto se $\left.\mathrm{Ti}<\mathrm{K}) ; 3^{\mathrm{a}}\right)\left\{\mathrm{x}_{\mathrm{it}}\right\}$ estritamente exógena com relação a $\left\{\mathrm{u}_{\mathrm{it}}\right\}$, o que implica em dizer que a $\operatorname{cov}\left(\mathrm{u}_{\mathrm{it}}, \mathrm{x}_{\mathrm{is}}\right)=0$; $\left.4^{\mathrm{a}}\right)$ por enquanto, $\left\{\mathrm{u}_{\mathrm{it}}\right\}$ homocedástico e não autocorrelacionado.

No caso de painéis lineares, a estimativa dos coeficientes $\beta$ são inconsistentes pelo método dos Mínimos Quadrados Ordinários (MQO), na medida em que a heterogeneidade não observada ou efeitos individuais $(\eta i)$ podem estar correlacionados com os regressores e, portanto, teremos a $\operatorname{cov}(\eta i$, xit $) \neq 0$. 
Uma alternativa é o método do MQO empilhado (ou Pooled OLS), o qual assume que os regressores são exógenos e considera apenas a parte $u_{i t}$ do erro (WOOLDRIDGE, 2002). A especificação fica a seguinte, então, para o modelo pooled:

$$
\text { yit }=x^{\prime} \text { it } \beta+\text { uit }
$$

O método dos MQO empilhado (pooled OLS) apresenta erros padrões robustos por agrupamentos, o que corrige a variância dos indivíduos/grupos (within) ao longo do tempo. Esses estimadores pooled são consistentes se o modelo de efeito aleatório (RE) é apropriado, e são inconsistentes se o modelo de efeito fixo (EF) é apropriado.

Para tratamento dos efeitos individuais do erro composto $(\eta i)$, pode-se utilizar o efeito fixo (FE), que considera que $\eta i$ é constante, ou o efeito aleatório (RE), que adota que esse termo $\eta i$ é extraído independentemente de alguma distribuição de probabilidade.

No caso dos modelos lineares com efeitos fixos (FE), fixed effect models, são modelos de variáveis dummy, com termos constantes específicos por indivíduo. Assim sendo, temos que $E\left[\eta_{i} l X_{i}\right]=g\left(X_{i}\right)$ e, portanto, os efeitos individuais $\eta_{i}$ são correlacionados com as variáveis incluídas, sendo a $\operatorname{Cov}\left[\mathrm{x}_{\mathrm{it}}, \eta_{\mathrm{i}}\right] \neq 0$. Assume que os efeitos específicos por indivíduos $\left(\eta_{\mathrm{i}}\right)$ não variam com o tempo. Assim, os efeitos individuais $\left(\eta_{\mathrm{i}}\right)$ são tratados como parâmetros, com diferentes interceptos individuais, além de serem variáveis aleatórias correlacionadas com todos os regressores (WOOLDRIDGE, 2002).

Usando o método dos efeitos fixos, o componente dos efeitos individuais $\left(\eta_{i}\right)$ é eliminado. Assim, nesse caso dos efeitos fixos, a especificação do modelo de painéis lineares estáticos é a seguinte:

$$
\mathrm{y}_{\mathrm{it}}=\alpha_{\mathrm{i}}+\mathrm{X}^{\prime}{ }_{\mathrm{it}} \beta+\varepsilon_{\mathrm{it}}
$$

No modelo de efeito fixo (EF), o erro u_i é correlacionado com os regressores e, portanto, nesse modelo a corr $\left(\mathrm{u} \_\mathrm{i}, \mathrm{Xb}\right)$ é diferente de 0 . Considere que o sigma_u é a parte do erro devido a variança dos indivíduos e sigma_e é a parte do erro devido a variança do erro aleatório. O rho é a proporção da variança total devido a variança dos indivíduos, é a correlação intra-classe. O teste de $\mathrm{F}$ usado nesse modelo testa que todos os u_i são iguais a zero. Se não aceitou a hipótese H0, o rho estabelece a fração da variança que é devido ao u_i. 
Um modelo de efeito fixo trata os efeitos individuais $\left(\eta_{\mathrm{i}}\right)$ como uma variável aleatória que pode ser correlacionada com os regressores $x_{i t}$. Em painéis longos (com muitos períodos de tempo e relativamente poucos indivíduos) isto não representa nenhum problema. Mas em painéis curtos (com muitas unidades individuais observadas e poucos períodos de tempo), a estimação conjunta de $\eta_{i}$ e outros parametros geralmente leva a estimação inconsistente de todos os parâmetros.

Por outro lado, nos modelos lineares com efeitos aleatórios (RE), randon effect models, são entendidos como modelos de componentes do erro, na medida em que temos $\mathrm{E}\left[\eta_{\mathrm{i}} \mid \mathrm{X}_{\mathrm{i}}\right]=\mu$, os efeitos individuais $\left(\eta_{\mathrm{i}}\right)$ não são correlacionados com as variáveis explicativas incluídas e, portanto, a $\operatorname{Cov}\left[\mathrm{x}_{\mathrm{it}}, \eta_{\mathrm{i}}\right]=0$. Um modelo $\mathrm{RE}$ trata o efeito individual específico $\left(\eta_{\mathrm{i}}\right)$ como uma variável aleatória não observada com uma distribuição especificada, em geral a distribuição normal. Nesses casos de modelos lineares com efeitos aleatórios, a especificação é a seguinte:

$$
\text { yit }=x^{\prime} \text { it } \beta+\text { cit }+ \text { uit }
$$

Nos modelos de efeitos aleatórios (RE), deve-se ressaltar que se assume que a $\operatorname{corr}\left(\mathrm{u} \_\mathrm{i}, \mathrm{X}\right)=0$. Esse modelos de painéis lineares estáticos com RE devem ser estimados pelo método MQG (Mínimos Quadrados Generalizados) ou pelo MQO empilhado (ou pooled), que geram estimadores consistentes (WOOLDRIDGE, 2002).

Voltando a seguinte especificar esse modelo da seguinte forma:

$$
\begin{gathered}
y_{i t}=x^{\prime}{ }_{i t} \beta+\varepsilon_{i t} \\
\text { sendo: } \varepsilon_{i t}=\eta_{i}+u_{i t}
\end{gathered}
$$

Pode-se fazer a seguinte transformação:

$$
\mathrm{y}_{\mathrm{it}}-\overline{\mathrm{y}}_{\mathrm{it}}=\beta^{\prime}\left(\mathrm{x}_{\mathrm{it}}-\mathrm{x}^{-}{ }_{\mathrm{i}}\right)+\varepsilon_{\mathrm{it}}-\varepsilon^{-}{ }_{\mathrm{i}}
$$

Essa transformação é denominada de within, e gera os estimadores within, que se baseiam em variáveis intra-individuais, e não entre indivíduos. A variação within é a soma dos quadrados das diferenças entre a observação de cada individuo e a sua média de cada individuo. Os estimadores within são os estimadores de efeito fixo (FE).

Há outro estimador que usa somente a informação sobre as médias individuais, é o denominado estimador between. A variação between é a soma dos quadrados das diferenças entre as médias de cada individuo e a média de toda a amostra. A especificação fica da seguinte forma: 


$$
\bar{y}_{i}=\beta^{\prime} x^{-}{ }_{i}+\varepsilon^{-}{ }_{i}
$$

Destaca-se que o modelo de efeitos aleatórios (RE) é uma combinação do estimador de efeitos fixos (within) e do estimador between. Esse é o estimador global, que é uma média ponderada dos estimadores within e between, com os pesos corretos.

A questão que fica é como escolher entre os modelos FE ou RE. O teste de Hausman (1978) é apropriado para essa escolha, na medida em que esse teste consiste em verificar se a diferença entre o FE e o RE é estatisticamente significativa. O modelo FE é considerado preferível devido ao fato dele permitir a correlação entre os efeitos individuais $\left(\eta_{\mathrm{i}}\right)$ e as variáveis explicativas.

A hipótese nula H0 considera que a diferença entre os coeficientes estimados por EF (within) e RE não é sistemática. O Teste de Hausman assume que o estimador de efeitos aleatórios é eficiente sob a hipótese nula $\mathrm{H} 0$ e inconsistente sob a hipótese alternativa Ha, e que o estimador de efeitos fixos é consistente sob a hipótese $\mathrm{H} 0$ e Ha.

Se o teste de Hausman mostrar que a diferença é significativa (Prob $>\operatorname{chi} 2<0,05$ ), não se aceita H0. Assim, ficamos com o EF, já que a diferença entre os coeficientes estimados por EF (within) e RE é significativa. Nesse caso, o RE não é consistente e foi rejeitado, em favor do EF. Em síntese, é o teste de Hausman que vai verificar se a diferença entre esses modelos é significativa. Se for, tem que usar o EF(WOOLDRIDGE, 2002).

Caso contrário, de acordo com o teste de Hausman, se não se rejeita a hipótese nula H0, o estimador RE é eficiente e, portanto, deve-se utilizar o modelo RE. Nesse caso, é possível verificar pelo teste de Hausman que não há diferença sistemática entre os dois modelos e, portanto, não se rejeita a hipótese nula, optando-se pelo modelo RE.

\section{4 - RESULTADOS E DISCUSSÕES}

A unidade de análise corresponde aos vinte e sete municípios componentes do Vale do Mucuri, localizado em Minas Gerais, a saber: Ataléia, Bertópolis, Campanário, Caraí, Catuji, Frei Gaspar, Fronteira dos Vales, Itaipé, Itambacuri, Ladainha, Ouro Verde de Minas, Pescador, Santa Helena de Minas, Setubinha, Águas Formosas, Carlos Chagas, Crisólita, Franciscópolis, Machacalis, Malacacheta, Nanuque, Novo Oriente de Minas, Pavão, Poté, Serra dos Aimorés, Umburatiba e Teófilo Otoni (Figura 1). 


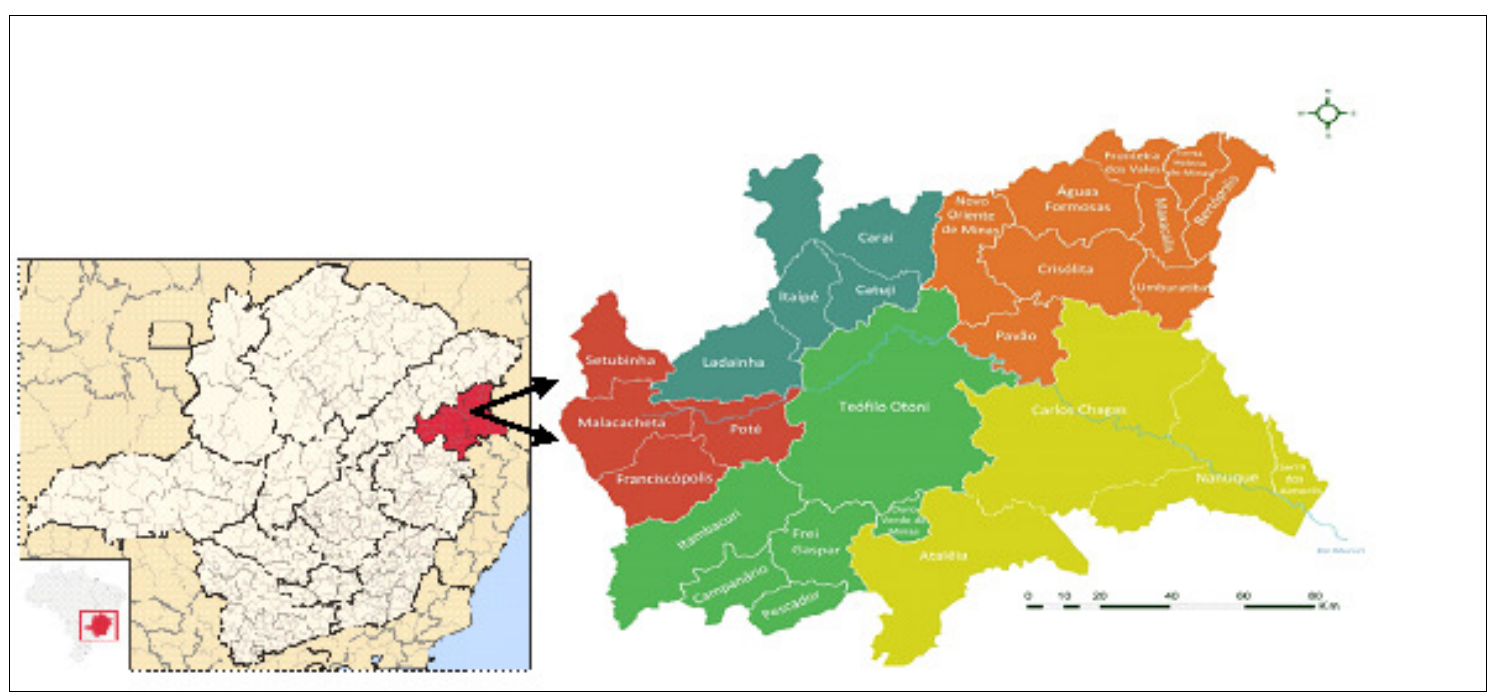

Figura 1 - Vale do Mucuri, Minas Gerais. Fonte: GEPAF - Grupo de Extensão e Pesquisa em Agricultura Familiar, Universidade Federal dos Vales do Jequitinhonha e Mucuri (UFVJM), 2010.

O Vale do Mucuri, o qual possui uma área de aproximadamente $23.2 \mathrm{mil} \mathrm{km}{ }^{2}$, totaliza uma população de mais de 438 mil habitantes (IBGE, 2010), representando, aproximadamente, 2,4\% da população do estado de Minas Gerais. Teófilo Otoni é o município de maior população do território, com cerca de 135 mil habitantes, enquanto Umburatiba apresenta o menor conjunto populacional, com cerca de 2.700 habitantes. $\mathrm{O}$ Índice de Desenvolvimento Humano médio é de 0.68.

O grau de urbanização dos dois municípios mais populosos (Teófilo Otoni e Nanuque), é de, aproximadamente, 66\% (IBGE, 2010). Descontados esses dois municípios mais populosos, os 25 municípios restantes apresentam uma população total média de cerca de 10 mil habitantes por município.

Trata-se de uma das regiões mais pobres de Minas Gerais, apresentando, em geral, baixa renda per capita e baixo Índice de Desenvolvimento Municipal, comparados às demais regiões do Estado. Os municípios são marcadamente rurais e esta região caracteriza-se por uma menor expressão econômica em comparação com as demais áreas do Estado.

No que diz respeito às finanças públicas da região, o trabalho de Silva, Wakim \&Wakin(2014) aponta para um déficit de accountability, isto é, ainda está limitado o cumprimento das exigências legais de publicidade, transparência e participação no que tange aos planos e resultados da gestão local. Leite Filho et al (2016) apontam para situação difícil quanto à posição fiscal, em virtude do elevado gasto com pessoal e falta de liquidez desses municípios. A (falta de) qualidade da gestão fiscal se relacionaria não somente à má administração mas às limitadas oportunidades de arrecadação presentes. 
A variação média do indicador receita tributária/receita corrente pode ser visualizada no Gráfico 1 a seguir. Pode ser observada uma queda no indicador para os períodos eleitorais.

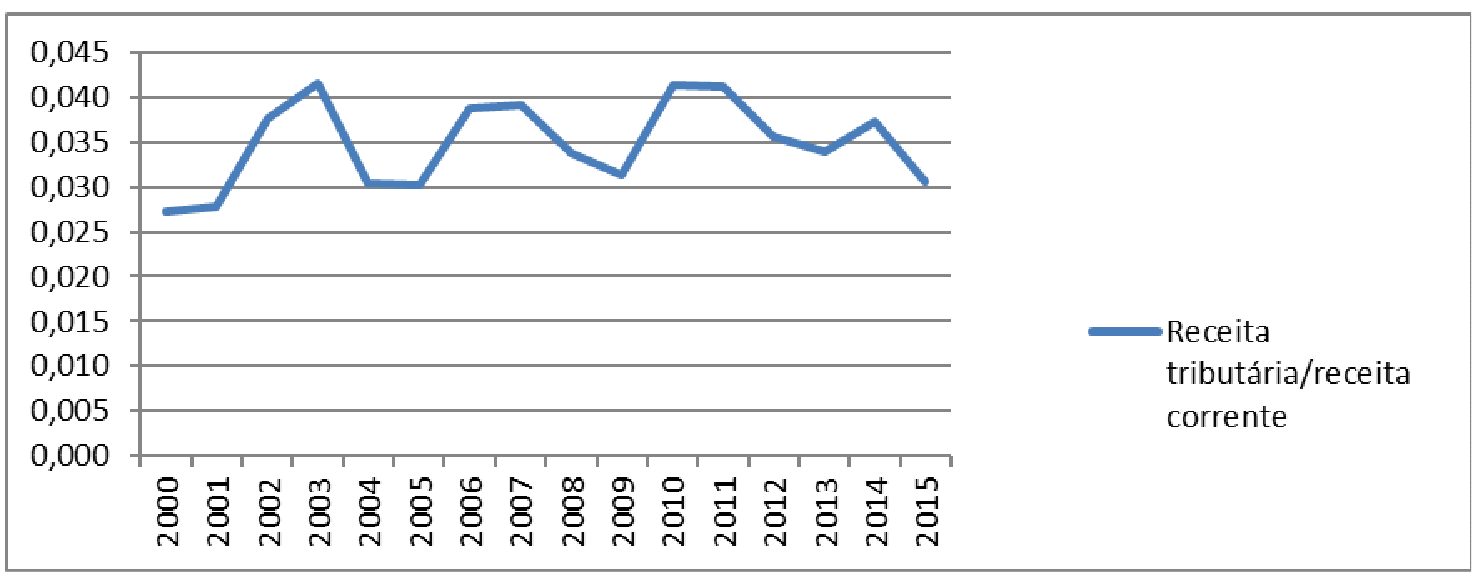

Gráfico 1 - Indicador Receita tributária/receita corrente - Vale do Mucuri.

Fonte: Elaboração própria com base nos dados Finbra.

O Gráfico 2 disponibiliza informações sobre o indicador gastos com pessoal/receita corrente. O referido indicador por município do Vale do Mucuri pode ser acompanhado no Anexo 1.

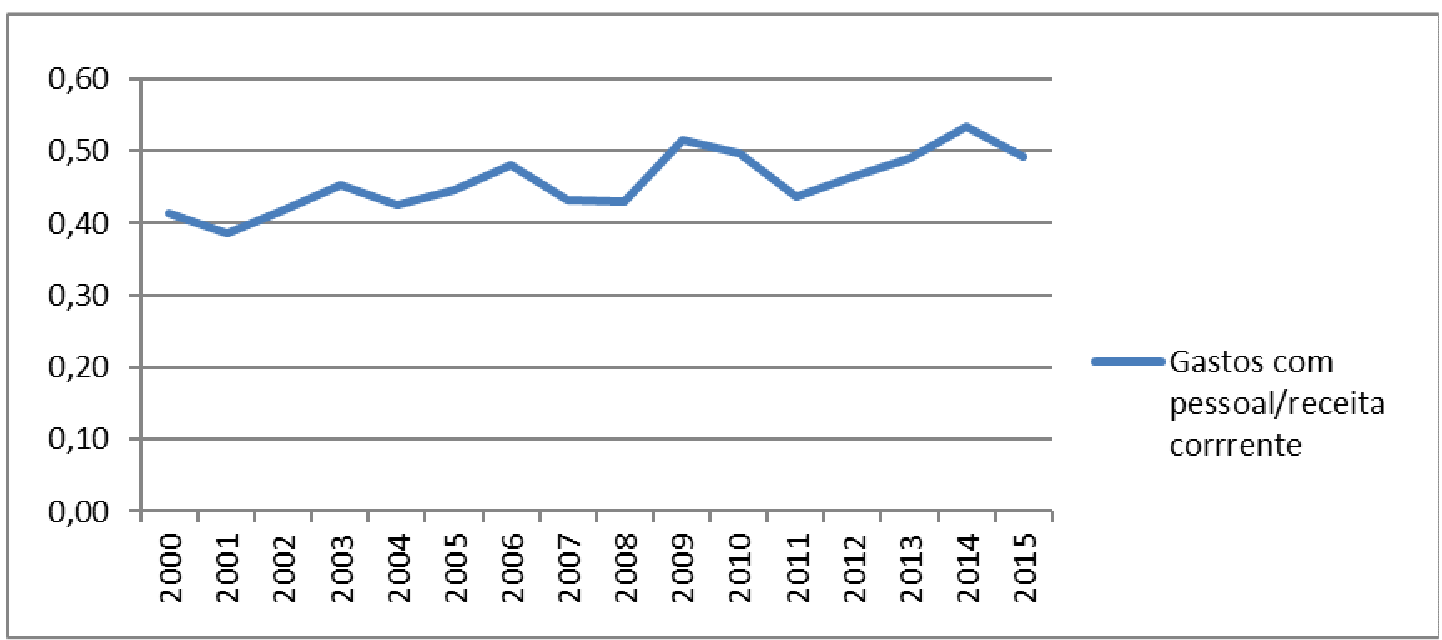

Gráfico 2 - Indicador gastos com pessoal/receita corrente - Vale do Mucuri. Fonte: Elaboração própria com base nos dados Finbra.

De acordo com o Gráfico 2, percebe-se uma elevação da média do indicador gastos com pessoal/receita corrente ao longo do período considerado, com maiores valores para os anos 2009 e 2014.

Os indicadores que apresentaram maior correlação com resultado primário per capita foram receita tributária/receita corrente (correlação negativa), gastos com pessoal/receita corrente (correlação negativa) e a Dummy1 (correlação positiva), 
respectivamente. A correlação negativa do indicador receita tributária/receita corrente sugere que a receita tributária não tem sido componente expressivo da receita corrente, possivelmente dada a limitação de arrecadação presente nos municípios do Vale do Mucuri.

Os resultados dos modelos lineares de dados em painel podem ser observados na Tabela 1 a seguir.

Tabela 1- Resultados dos modelos de painel linear - Vale do Mucuri

\begin{tabular}{|c|c|c|c|c|}
\hline $\begin{array}{l}\text { Resultado primário per } \\
\text { capita }\end{array}$ & Pooled & $\begin{array}{c}\text { Efeitos } \\
\text { fixos }\end{array}$ & Between & $\begin{array}{l}\text { Efeitos } \\
\text { aleatórios }\end{array}$ \\
\hline $\begin{array}{l}\text { Despesas com } \\
\text { pessoal/receita } \\
\text { corrente liquida }\end{array}$ & $\begin{array}{c}-1509.768 \\
(470.5466) \\
0.004 \\
\end{array}$ & $\begin{array}{c}-2166.276 \\
(565.8377) \\
0.000 \\
\end{array}$ & $\begin{array}{c}-1265.845 \\
(530.6437) \\
0.025 \\
\end{array}$ & $\begin{array}{c}-1684.777 \\
(382.4344) \\
0.000 \\
\end{array}$ \\
\hline $\begin{array}{l}\text { Receita } \\
\text { tributária/Receita } \\
\text { corrente liquida }\end{array}$ & $\begin{array}{c}-457.6402 \\
(157.8007) \\
0.007 \\
\end{array}$ & $\begin{array}{c}-436.3619 \\
(74.30257) \\
0.000 \\
\end{array}$ & $\begin{array}{c}-489.356 \\
(206.3272) \\
0.026 \\
\end{array}$ & $\begin{array}{c}-449.9309 \\
(69.54342) \\
0.000 \\
\end{array}$ \\
\hline Dummy 1 & $\begin{array}{c}206.3404 \\
(21.4787) \\
0.000 \\
\end{array}$ & $\begin{array}{c}211.0795 \\
(29.38504) \\
0.000 \\
\end{array}$ & - & $\begin{array}{c}207.5003 \\
(29.15904) \\
0.000 \\
\end{array}$ \\
\hline Dummy 2 & $\begin{array}{c}-184.799 \\
(20.92432) \\
0.000\end{array}$ & $\begin{array}{c}-188.5276 \\
(29.58474) \\
0.000\end{array}$ & - & $\begin{array}{c}-185.6348 \\
(29.37828) \\
0.000\end{array}$ \\
\hline Constante & $\begin{array}{c}203.5912 \\
(67.59389) \\
0.006 \\
\end{array}$ & $\begin{array}{c}212.6338 \\
(41.71079) \\
0.000\end{array}$ & $\begin{array}{c}391.4254 \\
(91.17876) \\
0.000\end{array}$ & $\begin{array}{c}205.1593 \\
(40.58596) \\
0.000\end{array}$ \\
\hline
\end{tabular}

Fonte: Elaboração Própria.

Os modelos estimados foram: método dos MQO empilhado (pooled OLS), com erros padrões robustos por agrupamentos, de modo a corrigir a variância dos indivíduos/grupos (within) ao longo do tempo; modelo de efeitos fixos; modelo de efeitos aleatórios e estimador between.

Considerando o método pooled OLS, foram significativos e negativos os indicadores: Despesas com pessoal/receita corrente liquida, Receita tributária/Receita corrente liquida, e Dummy2. E foi significativo e diretamente proporcional ao resultado primário per capita o indicador Dummy1. Os resultados das estimações evidenciaram que o resultado primário per capita pode ser explicado em $25 \%$ pelo conjunto dos indicadores utilizados. A estimação foi significativa a 0,01 (teste F) e foram observados coeficientes significativos a 0,01 para todas as variáveis do modelo de regressão, sendo os sinais dos parâmetros coerentes com as expectativas a priori, confirmando os resultados da matriz de correlação.

Para o modelo de efeitos fixos, o resultado primário é inversamente proporcional aos indicadores Despesas com pessoal/receita corrente liquida, Receita tributária/Receita corrente liquida, e Dummy1. Para a variável Dummy1, o efeito é 
positivo, implicando em um aumento de cerca de $\mathrm{R} \$ 206,34$ por habitante no resultado primário per capita, conforme os anos analisados. A estatística do teste $\mathrm{F}$, bem como seu respectivo p-valor indica que o modelo de efeitos fixos é preferível ao pool (Prob $>\mathrm{F}=$ $0.0004)$.

No modelo between, foram significativos os regressores Despesas com pessoal/receita corrente liquida, Receita tributária/Receita corrente liquida. $\mathrm{O}$ indicador Despesas com pessoal/receita corrente reduz o resultado primário (1265.84) e o indicador Receita tributária/Receita corrente liquida é inversamente proporcional ao resultado primário conforme considerado (489.356).

Para o modelo de efeitos aleatórios, os regressores Despesas com pessoal/receita corrente liquida (-1684.777), Receita tributária/Receita corrente liquida(-449.9309) e Dummy2 (-185.6348) são inversamente relacionados ao resultado primário per capita no período de 2000 a 2015. Todos os indicadores foram significativos a $1 \%$. A variável Dummy1, positivamente significativa, eleva em $\mathrm{R} \$ 207,50 /$ habitante o resultado primário per capita. Os erros padrão são menores do que os computados para a estimação within, em virtude da utilização de variação between.

A partir da realização do teste de Hausman, verificou-se que o estimador de efeitos aleatórios é eficiente (Prob>chi2 $=0.8533$ ). Nesse caso, é possível verificar pelo teste de hausman que não há diferença sistemática entre os modelos e, portanto, não se rejeita a hipótese nula.

Desse modo, para os municípios do Vale do Mucuri, há efeitos significativos entre os indicadores utilizados, com respaldo na LRF e o resultado primário per capita municipal. Pôde ser observado que as despesas com pessoal em termos da receita corrente possuem impacto negativo sobre o resultado primário, assim como as receitas tributárias, em termos da receita corrente. Além disso, foi observado que o impacto das despesas com pessoal é maior que o impacto das receitas tributárias sobre o resultado primário. Tais resultados apontam para uma necessidade de gestão dos gastos com pessoal mais efetiva e respaldada na LRF, assim como da necessidade de um maior dinamismo na arrecadação de receitas tributarias por parte desses municípios.

\section{5- CONSIDERAÇÕES FINAIS}

O objetivo desse trabalho foi analisar os impactos da Lei de Responsabilidade Fiscal no comportamento fiscal dos municípios do Vale do Mucuri, nos anos 2000 a 
2015, a partir da utilização de dados em painel. Para tanto, foram utilizados os indicadores despesa de pessoal/receita corrente e receita tributária/receita corrente, procurando-se verificar quais fatores de natureza fiscal determinam a probabilidade de um município obter resultados primários positivos ou negativos.

Os resultados apresentados fornecem subsídios para melhor compreensão da variação do resultado primário per capita nos municípios do Vale do Mucuri ao longo do período de análise. Caracterizados, de modo geral, por seu porte pequeno, baixo dinamismo de suas economias e indicadores sociais de baixo desempenho, esses municípios apresentam, quanto às suas finanças, pouca transparência de informações, receitas tributárias limitadas e gastos com pessoal elevados.

Por intermédio da estimação do modelo de dados em painel foi possível explorar a dimensão temporal do resultado primário per capita. Em conformidade com testes realizados, o modelo global de efeitos aleatórios apresentou-se como mais apropriado. Evidenciou-se a relação negativa entre o resultado primário, conforme estimado, e as relações gastos com pessoal/receita corrente e receitas tributárias/receita corrente. Além dessa, detectou-se a relação negativa entre a Dummy2 e a variável dependente, apontando para o fato de que no ano 2001 possivelmente esses municípios não atendiam as especificações da LRF. O indicador Dummy1 apresentou relação positiva e significativa com o resultado primário, possivelmente indicando que, após o ano de 2001, os municípios do Vale do Mucuri melhoraram sua capacidade de atendimento às especificações da referida Lei.

Como sugestões para estudos futuros sugere-se a continuidade desse trabalho, de modo a acompanhar os indicadores analisados, assim como, ampliar a pesquisa de modo a contemplar os municípios do Vale do Jequitinhonha. Ademais, ressalta-se a relevância de se adicionar indicadores como, proximidade a centros urbanos médios, representação política, assim como indicadores demográficos e econômicos, de maneira a se ter uma análise mais completa.

\section{REFERÊNCIAS}

ANDRADE, N. A. Contabilidade pública na gestão municipal. 3a ed. São Paulo: Atlas, 2007.

BOTELHO, Milton Mendes. Manual prático de controle interno na administração pública municipal. Curitiba: Juruá, 2007.

BRASIL. Constituição. Constituição da República Federativa do Brasil. Brasília, DF: Senado Federal: Centro Gráfico, 1988. 292 p. 
BRASIL. Lei Complementar $n^{\circ}$. 101, de 4 de maio de 2000. Estabelece normas de finanças públicas voltadas para a responsabilidade de gestão fiscal e dá outras providências.

BRASIL. IBGE - Instituto Brasileiro de Geografia e Estatística. Municípios brasileiros. Disponível em: www.ibge.gov.br. Acesso em 10 de junho de 2017.

Cameron, A.C., Trivedi, P.K. Microeconometrics: Methods and Applications. Cambridge University Press, 2005.

Cameron, A.C., Trivedi, P.K. Microeconometrics Using Stata. Texas Solution, 2009.

CARVAlHO, Deusvaldo. Orçamento e Contabilidade Pública. São Paulo: Campus, 2010.

FIORAVANTE, D. G.; PINHEIRO, M. M. S.; VIEIRA, R. S. V.; SANTOS, J. C. Lei de responsabilidade fiscal e finanças públicas municipais: impactos sobre despesas com pessoal e endividamento. IPEA, 2006. (Texto para Discussão, n. 1223).

GERIGK, W.O impacto da Lei de Responsabilidade Fiscal sobre a gestão financeira de pequenos municípios. Dissertação do Programa de Mestrado em Contabilidade Universidade Federal do Paraná, Curitiba, 2008.

LEITE FILHO, G. A. \& Fialho, T. M. M. Associação entre Indicadores de Gestão Pública municipal e Indicadores de Desenvolvimento dos Municípios Brasileiros. In: XIV CONGRESSO USP DE CONTROLADORIA E CONTABILIDADE, 14. São Paulo. FEA/USP, 2014.

LEITE FILHO, G. A. ; Martins, F. S. A. ; Alves, F. O. ; Alves, J. A. R. . Indicadores de qualidade da gestão fiscal pública dos municípios da mesoregião norte de minas. In: XVII Seminário sobre a Economia Mineira, 2016, Diamantina. Anais do XVII Seminário sobre a Economia Mineira, 2016. v. 1. p. 1-15.

MARTINS, I. G. S.; NASCIMENTO, C. V. Comentários à lei de responsabilidade fiscal. 2. ed. São Paulo: Saraiva, 2007.

MATIAS, AB e CAMPELLO, CAGB. Administração financeira municipal. São Paulo: Atlas, 2000.

MENEZES, A. M. F. (2002). O processo de descentralização e as contas públicas: um estudo sobre as bases financeiras municipais baianas. 2002. $188 \mathrm{f}$. Tese (Doutorado em Administração). Curso de pós-graduação em administração, Universidade Federal da Bahia, Salvador.

MENEZES, R. T. Impactos da lei de responsabilidade fiscal sobre os componentes de despesa dos municípios brasileiros. Brasília: ESAF, 2005. 64 p. Monografia premiada em $1^{\circ}$ lugar no X Prêmio Tesouro Nacional - 2005, Lei de Responsabilidade Fiscal, Brasília (DF). 
MOURA NETO, J. S.; PALOMBO, P. E. M. Lei de responsabilidade fiscal e a arrecadação própria dos municípios brasileiros. In: ENCONTRO ANUAL DA ASSOCIAÇÃO NACIONAL DOS PROGRAMAS DE PÓS-GRADUAÇÃO EM ADMINISTRAÇÃO, 30, XVIII Congresso Brasileiro de Custos - Rio de Janeiro - RJ, Brasil, 07 a 09 de novembro de 2011 2006, Salvador.

NASCIMENTO, E. R.; DEBUS, I. Lei complementar $n^{o}$. 101/2000: entendendo a Lei de Responsabilidade Fiscal. 2011.

OLIVEIRA, F. H.; PETER, M. G. A.; MENESES, A. F. Lei de Responsabilidade Fiscal: implicações nos indicadores sociais municipais. In: CONGRESSO USP DE CONTROLADORIA E CONTABILIDADE, 10, 2010. Disponível em: . Acesso em: 25 jun. 2017.

SANTOLIN, Roberto; JAYME JR., Frederico G.; REIS, Júlio César dos. Lei de Responsabilidade Fiscal e Implicações na Despesa de Pessoal e de Investimento nos Municípios Mineiros: Um Estudo com Dados em Painel Dinâmico. Revista Estudos Econômicos, v.39, n.4, p. 895-923. São Paulo, 2009.

SAKURAI, SN. Déficit e ajuste fiscal dos municípios paulistas: uma análise para o período 1989 - 2001 via dados em painel. Revista Economia, Brasília, v.6, n.2, p.463488, jul/dez 2005.

SILVA, S., WAKIM, V., \& WAKIM, E. 2014 Mar 6. A transparência da gestão pública dos municípios do vale do Mucuri (MG)- uma síntese do Déficit de Accountability. Revista Brasileira de Contabilidade. [Online] :205

SOUSA, P. F. B., GOMES, A. de O., LIMA, A. O., PETER, M. da G. A., \& MACHADO, M. V. V. (2011). Lei de responsabilidade fiscal e sua relação com o desenvolvimento municipal: Uma análise utilizando dados em painel. Anais do Congresso Brasileiro de Custos - CBC, Rio de Janeiro, Brasil, 18.

VELOSO, Gilberto O.; TEIXEIRA, Anderson M. A Lei de Responsabilidade Fiscal e as microrregiões do estado do Rio Grande do Sul: uma análise empírica. Ensaios FEE, Porto Alegre, v. 28, n. 2, p. 443-470, out. 2007.

WOOLDRIDGE, J. M. Econometric Analysis of Cross Section and Panel Data. Cambrigde and London: MIT Press, 2002. 